
allemande

51-2| 2019

Les Humanités environnementales : circulations et renouvellement des savoirs en France et en Allemagne

\title{
Zur Konstruktion „humanimaler" Emotionen in Otto Alschers Die Bärin. Natur- und Tiergeschichten aus Siebenbürgen
}

Stefan Hecht

\section{OpenEdition}

\section{Journals}

Édition électronique

URL : https://journals.openedition.org/allemagne/1992

DOI : 10.4000/allemagne.1992

ISSN : 2605-7913

Éditeur

Société d'études allemandes

Édition imprimée

Date de publication : 10 décembre 2019

Pagination : 371-386

ISSN : 0035-0974

Référence électronique

Stefan Hecht, "Zur Konstruktion "humanimaler“ Emotionen in Otto Alschers Die Bärin. Natur- und Tiergeschichten aus Siebenbürgen", Revue d'Allemagne et des pays de langue allemande [Online], 51-2 I 2019, Online erschienen am: 02 Dezember 2020, abgerufen am 19 Mai 2021. URL: http:// journals.openedition.org/allemagne/1992 ; DOI: https://doi.org/10.4000/allemagne.1992 


\section{Zur Konstruktion „humanimaler" Emotionen in Otto Alschers Die Bärin. Natur- und Tiergeschichten aus Siebenbürgen}

- Stefan Hecht*

\section{Einleitung}

Seit wenigen Jahrzehnten ist von einem animal turn die Rede. Lebenswissenschaften und Geisteswissenschaften lenken vermehrt ihren Fokus auf Tiere. Ein eigenes Forschungsfeld, die Human Animal Studies (HAS) ${ }^{(1)}$, hat sich formiert. Nicht nur in der Quantität, auch in der Qualität der Aufmerksamkeit spiegeln sich neue Prämissen, durch die Tiere vom Objekt der Beobachtung zum Subjekt mit intrinsischen Rechten rücken. Faktoren für diesen ethischen Bewusstseinswandel sind einerseits die vermehrte Anhäufung von Wissen über Tiere, deren Komplexität und Emotionen, wodurch die Grenze zwischen Mensch und Tier instabil geworden ist, und andererseits das Ausmaß der Industrialisierung von Tieren. Animal studies berühren mitunter ein anderes Forschungsfeld, namentlich dann, wenn es um Wildtiere geht: Die Environmental studies ${ }^{(2)}$.

Otto Alscher (1888-1944) wurde im Banat, einer deutschsprachigen Region der Donaumonarchie, die seit 1918 zu Rumänien gehört, geboren. Er lebte als Journalist und Schriftsteller in Wien und Budapest und zuletzt im Banat. 1944, nach der

* Stefan Hecht ist seit 2016 Doktorand an der Universität Straßburg, stefan.hecht2@etu.unistra.fr.

1 Einen Pioniercharakter hat Peter Singers Werk Animal Liberation von 1975. Manche datieren den Beginn der HAS als eigenes Forschungsfeld mit der ersten Ausgabe der Zeitschrift Anthrozöos 1987. Folgende Werke können einen Überblick über die aktuellen HAS geben: Roland Borgards (Hg.), Tiere: Kulturwissenschaftliches Handbuch, Stuttgart, J.B. Metzler, 2016. Einen Eindruck über den status quo der Forschungslage in Frankreich kann folgender Artikel vermitteln: Nicolas Delon, „Études animales: un aperçu transatlantique“, Tracés, Revue des sciences humaines, Hors-Série, 15 (2015), traces.revues.org/6274.

2 Perspektiven einer Interdisziplinarität von Umwelt- und Tierestudien im 21. Jahrhundert weist: Aurélie Choné, Isabelle Hajek und Philippe Hamman (Hg.), Rethinking Nature. Challenging Disciplinary Boundaries, London/New York, Routledge, 2017. 
Kriegserklärung Rumäniens an das Deutsche Reich, wurde er als Angehöriger einer deutschen Volksgruppe von der Roten Armee in ein Lager interniert, wo er starb. Alscher engagierte sich für eine Aufrecherhaltung der deutschsprachigen Identität seiner Heimat. Wildtiere sind in seinem Gesamtwerk ein wiederkehrendes, zentrales Motiv und bilden ein Gegengewicht zu einem anthropozentrischen Weltbild.

Der Band Die Bärin. Natur- und Tiergeschichten aus Siebenbürgen wurde erstmals $1943^{(3)}$ verlegt. Er umfasst 19 eigenständige Geschichten, die um 1940 entstanden sind. Manche sind mehr kontemplativer Art, andere eher abenteuerlich. Die landschaftliche Kulisse bilden die rumänischen Karpaten. Die Wildtiere haben im Allgemeinen den Status einer Person mit individuellen Zügen und manche der Geschichten werden vom Erzähler aus der Perspektive der Tiere erzählt. Die Erscheinung der Wildtiere, auch wenn es Bären oder Wölfe sind, die ja in den Karpaten existierten, ist von einem realistischen Charakter. In jüngerer Vergangenheit publizierten die Germanistin Helga Korodi und Axel Goodbody (ecocriticism) über den Autor ${ }^{(4)}$. Forschungsgegenstand der literarischen Animal studies ist Alscher bisher allerdings noch nicht gewesen. Der Titel mit dem Ausdruck humanimal verweist nicht nur auf die Präsenz von Tieren und Menschen und Emotionen, sondern auch auf deren Verschränktsein ${ }^{(5)}$. Menschen, Wildtiere und deren Emotionen hängen irgendwie zusammen. Das gegenseitige Berühren, Sehen, Interferieren von Wildtieren und Menschen geht mit sich wechselseitig bedingenden Emotionen einher.

Der Aufsatz konzentriert sich auf Aspekte der Konstruktion von Emotionen zwischen Wildtieren und Menschen ${ }^{(6)}$. Bei der Lokalisierung der Emotionsebene sollen hier Autor (Produktion, Kontext) und Leser (Rezeption) außen bleiben und die textuelle Ebene, auf welcher Emotionen gebildet werden, ins Visier genommen werden. Grundsätzlich wird davon ausgegangen, dass das Medium Text insofern von Interesse bei der Betrachtung von Emotionsgenerierung ist, als der Modus der Narration als eine Art Schlüssel für emotionale, interpersonale Grundvorgänge begriffen werden kann ${ }^{(7)}$. Bei der Analyse erzählerischer, struktureller, sprachlicher und inhaltlicher Mittel, die beim Emotionsaufbau mitfungieren, werden durch die Benennung der Emotionen deren Rollen ad hoc angeschnitten, sollen hier jedoch zunächst nicht vordergründig behandelt werden. Der Artikel rekurriert auf eine Studie der Psychologie, die über die

3 Helga Korodi (Hg.), Die Bärin. Natur- und Tiergeschichten aus Siebenbürgen. Otto Alscher, Rangsdorf, Natur und Text, 2000. Der ursprüngliche Titel lautete: Die Bärin. Besinnliche Tiergeschichten. Das „Siebenbürgen“ in der Neuauflage ist irreführend, bei einer Ortsangabe wäre Banat zutreffender.

4 Axel Goodbody, „The Hunter as Nature-Lover: Idyll, aggression and ecology in the German animal stories of Otto Alscher", in: Fiona Becket und Terry Gifford (Hg.), Culture, Creativity and Environment. New Environmentalist Criticism, Amsterdam/New York, Rodopi, 2007, S. 135-159; Helga Korodi, „Die Täuschungen der Wildnis. Der Roman ,Zwei Mörder in der Wildnis“ von Otto Alscher“, Südostdeutsche Vierteljahresblätter, 52/2 (2003), S. 135-140.

5 Von einer „humanimalen Ästhetik“ spricht beispielsweise Susanne Schul angesichts der Verflechtung von Kriterien bei der vergleichenden Bewertung von Tieren und Menschen: Susanne Schul, Humanimale Ästhetik. Semantik und Narrativik ästhetischer Tier-Mensch-Relationierung, Göttingen, V\&R unipress, 2018.

6 Über gegenwärtige Emotionsforschung allgemein gibt Rüdiger Schnell einen umfassenden und kritischen Überblick: Rüdiger Schnell, Haben Gefühle eine Geschichte? Aporien einer History of emotions, Göttingen, V\&R unipress, 2015.

7 Fritz Breithaupt, Kulturen der Empathie, Frankfurt, Suhrkamp, 2013. 
Evaluierung emotionaler Gehalte in Wörtern den Bedarf emotionaler Dimensionen auslotet. Darauf aufbauend geht es darum, Emotionen in Alschers Geschichten „sichtbar“ zu machen. Dazu werden raumsemantische, kinetische und psychoakustische Momente untersucht. Anschließend konzentriert sich der Beitrag auf die Selektion zweier markanter Emotionen: moralische und existentielle Emotionen.

\section{Texte - Bausteine von Emotionen}

Seit der Einführung eines Dimensionen-Modells der Emotionen durch Wundt ${ }^{(8)}$ geht man in der Psychologie von der Notwendigkeit mindestens zweier Dimensionen aus, um Ähnlichkeiten bzw. Differenzen bei der Verarbeitung emotionaler Erfahrungen zu erfassen: valence (positiv versus negativ) und arousal (Erregungsaktivierung niedrig bis hoch).

Die Studie von Ellsworth, Fontaine et al..$^{(9)}$, deren Autoren auf dem Gebiet der Affective sciences profiliert ${ }^{(10)}$ sind, erfüllt die für die Psychologie typischen methodischen Ansprüche der Effizienz und der quantitativen Messbarkeit. Während literaturwissenschaftliche Emotionsforschung tendenziell text- und kontextsensitive Ansätze verfolgt, sensibilisiert o.g. Studie für eine Nahsicht auf die Einheiten, auf die Bausteine in der Rezeption und Produktion textlicher Emotionen ${ }^{(11)}$. Sie geht, abhängig von der jeweiligen Forschungsfrage, von der Notwendigkeit aus, ein Modell mit (mindestens) vier emotionalen Dimensionen zu applizieren. Es sollen, neben der Dimension (a) der qualitativen Bewertung eines Vorkommnisses und der Dimension (b) der potentiellen Erregungsquantität, die Dimension (c) der potentiellen Kontrolle und die Dimension (d) der Einschätzung bzw. Reaktion auf Unvorhergesehenes, Neues, berücksichtigt werden. Diese vier Dimensionen wurden von eigentlich sechs Komponenten (1. Bewertung, 2. psycho-physische Veränderungen, 3. motorischer Ausdruck, 4. Handlungstendenzen, 5. subjektive Erfahrungen, Gefühle, 6. Emotionsregulierung) auf vier komprimiert (PCA, principal component analysis, für einen optimalen „low dimensional space“). In der Versuchsanordnung wurden 531 Probanden 144 häufig in emotionaler Literatur vorkommende Wörter oder Satzfragmente zu einer Beund Auswertung gegeben. Die Worte bzw. Satzteile drücken eine Emotion aus, die zu den 24 in Wissenschafts- und Alltagssprache verwendeten Prototypen von Emotionen (wie Angst, Furcht, Glück, Hass, Verzweiflung usw.) einen Bezug haben. Das

8 Als Gründer des Instituts für experimentelle Psychologie wird Wilhelm Wundt mit dem Beginn naturwissenschaftlich-empirischer Psychologie gleichgesetzt. Mit der Konstruktion eines dimensionalen Modells von Emotionen versuchte Wundt um 1900 Emotionen wie Freude oder Angst in kleinere Bausteine zu zerlegen, bzw. zu erfassen, aus welchen „einfachen“ Gefühlen sich komplexere Gefühle zusammensetzen. Siehe Jochen Müsseler und Martina Rieger (Hg.), Allgemeine Psychologie, Berlin/Heidelberg, Springer, 2017, S. 186-194.

9 Phoebe C. Ellsworth, Johnny R.J. Fontaine, Etienne B. Roesch und Klaus R. Scherer, „The World of Emotions Is Not Two-Dimensional“, Psychological Science, 18/12 (2007), S. 1050-1057.

10 Klaus Scherer ist als Gründer des Swiss Center for Affective Sciences hervorzuheben.

11 Erwähnung findet das Dimensionen-Modell der Psychologie auch bei der Literaturwissenschaftlerin Simone Winko. Siehe Simone Winko, Kodierte Gefühle. Zu einer Poetik der Emotionen in lyrischen und poetologischen Texten um 1900, Berlin, Erich Schmidt Verlag, 2003, S. 74. Die recht verschiedenen Disziplinen wie Psychologie und Literaturwissenschaft bieten interessante Möglichkeiten gegenseitiger Befruchtung auf dem Gebiet von Text und Emotionen. 
Ergebnis dieser Studie bestätigt unter anderem die Erforderlichkeit von mehr als zwei emotionalen Dimensionen für die Repräsentation des semantischen Emotionsraums und erhellt die ähnliche Einschätzbarkeit bzw. Einschätzung mehrerer Personen von emotionalen Wörtern oder Satzteilen. Und sie legt ein Zurückgehen auf (reale) Erfahrungen nahe: „Although language is abstracted from known experience, it must correspond to human experience and represent important human concerns“(12).

Die Arbeitsparameter dieser Studie kongruieren mit der Hypothese einer gemeinsamen neurophysiologischen, sensorischen und motorischen Basis ${ }^{(13)}$ und eines kognitiv-kulturell kodierten Wissenspools von Emotionen und lassen sich mit einem diskursiven Ansatz literaturwissenschaftlicher Erforschung von Text und Gefühlen vergleichen, bei welchem eine Sprecherabsicht im Sinne der weit gefassten Ausdrucksthese $^{(14)}$ im Hintergrund bleibt.

Deshalb bleibt in diesem Teil, in dem es ja um die Konstruktion von Emotionen in Literatur geht, die Autorintentionalität ausgeklammert.

\section{Aspekte der Konstruktion von Emotionen in der Geschichte Über den Menschen}

Die erste Geschichte, Über den Menschen, handelt von der wenige Tage andauernden Episode im Leben eines Bären, kurz vor dessen geplantem Winterschlaf. Die Erzählung endet mit dem Tod des Bären. Berichtet wird in der 3. Person von einem heterodiegetischen Sprecher ohne Platz im Geschehen. Hauptfigur ist der Bär; Menschen ohne persönliches Profil treten auf bzw. werden erwähnt, des weiteren, auch ohne persönliches Profil, Haustiere, Vieh bzw. Ochsen; Meisen, Finken, Amseln, Geier, Raben, Wölfe, Füchse, ein Eichhörnchen, metaphorisch Maden, Gewürm, Raubtiere, sowie ein metaphorischer Mückenschwarm. Bereits im zweiten Satz wird eine Emotion des Bären benannt: „Eine Wanderlust war über ihn [den Bären, der im Herbst tiefer von

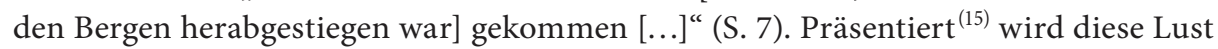

12 Ellsworth/Fontaine/Roesch/Scherer, „The World of Emotions“ (Anm. 9), S. 1056. Siehe Martin Koppenfels und Cornelia Zumbach (Hg.), Handbuch Literatur und Emotionen, Berlin/Boston, Walter de Gruyter, 2016: „Emotionsbegriffe stellen so gebietend wie kein anderes Sprachelement die Frage nach der Passung zwischen Wörtern und Dingen, das heißt in diesem Fall zwischen Wörtern und psychischer bzw. sozialer Erfahrung“, S. 6.

13 Aktuell immer performantere fMRI-Verfahren zeigen die Zusammenhänge und Prozesse zwischen emotionalem Gehalt von Wörtern und deren physiologischen Korrelationen. Siehe Benny BRIESEmeister, Arthur Jacobs, Lars Kuchinke et al., „Emotion in Reading: Dissociation of Happiness and Positivity“, Cognitive Affective \& Behavioral Neuroscience, 15/2 (2014), S. 287-298. In einer verlängerten Perspektive hinsichtlich eines spezifischen emotionalen Spektrums gegenüber Wildtieren wäre es z.B. nicht abwegig eine fMRI-Testreihen zu konzipieren und durchzuführen, die Leser (Betrachter) mit textuellen (respektive ikonographischen) Begegnungen von Wildtieren konfrontieren, um genauer analysieren zu können, welche morphologischen (über Kriterien und Nuancen des Kindchenschemas hinausgehende) Charakteristika von Tieren (wie Schlange, Kröte, Wildschwein, Reh, Eichhörnchen, Fuchs usw.) entsprechende Emotionen wecken.

14 D.h., dass man davon ausgehen kann, dass der vom Autor intendierte Emotionsausdruck mit seinem Sprachmaterial kongruiert, nicht aber dass der Autor und ein dahinterstehendes (Autor-) Gefühl übereinstimmen. Siehe S. Winko, Kodierte Gefühle (Anm. 11); Roland Barthes, „La mort de l'auteur“, Manteia, 5 (1968), S. 12-17.

15 Es ist inzwischen gängig zwischen der (impliziten) Ebene der Präsentation - sprachliche Mittel dienen als Verweise auf und Mittler von Emotionen - und der Thematisierung von Emotionen zu unterscheiden, bei welcher diese benannt werden. 
im Nebensatz desselbigen Satzes: „[...] er durchstreifte täglich weite Gebiete, durchquerte Wälder und schritt frei über einsame Waldwiesen. "Im dritten Satz vermindert der Sprecher das Absolute seines Wissens über den Bären, indem er den Satz mit „es war als“ beginnt: „Es war, als wollte er vor der tiefen Winterruhe nochmals die Einsamkeit der Wälder, die wilde Kraft seines Wanderns atmen, um dann in den Winterschlaf zu versinken, übersättigt und träumerisch." Dieser vagen Restriktion, was Wissen über und Beschaffensein von Sein und Emotionen des Bären angeht, folgen weitere. Es hat allerdings den Anschein, dass dadurch der Eindruck der Echtheit der Emotion des Tieres erhöht wird. „Noch einmal die tiefe Einsamkeit der Wälder, die wilde Kraft des Wanderns atmen, um dann übersättigt und träumerisch in den Winterschlaf zu sinken“: Von Anfang an rekurriert der Text auf den Lebensraum, mit den Topoi der Berge und der Wälder werden Vitalität, und mit übersättigt, träumerisch eine Emotion konnotiert, die als übergeordnete Kategorie positiv ist, als Basiskategorie für Freude steht, als untergeordnete Kategorie Zufriedenheit ausdrückt, aber als spezifischere Kategorie noch mehr involviert, nämlich eine höhere, zugleich mentale und physische Glückserfahrung, bei der der verbalisierte physiologische Atem metonymisch poetisiert wird.

In den ersten drei Sätzen wird die mit Emotionen verflochtene Substruktion der Geschichte gelegt: Ein Bär hat (nachvollziehbare und wichtig erscheinende) Bedürfnisse (Bewegung, Ruhe) bzw. Sehnsüchte, die mit dem Lebensraum Wald und mit dem Gefühl Einsamkeit verbunden sind. Doch dann lässt ein Geräusch den Frieden bedroht erscheinen: „Der Wald war voll Ruhe und Erfüllung. Zeitweise nur grollte es dumpf dort, wo der breite Strom die Berge durchschnitt und der Bär spürte, dass dort [...] ein Leben und Bewegen vieler Menschen war“ (S. 7). Mit dem Auftauchen der Menschen und des sonoren Phänomens im 5. Satz beginnt die Handlung von Flucht und Flüchtigen zu erzählen, und die zuvor präsentierte positive Emotion erhält ihren Gegenpol. Darauf folgt nun eine explizite Einschränkung der kognitiven Fähigkeiten des Bären vom Sprecher:

„Was es war und weshalb es geschah, wusste der Bär nicht, denn er hatte ja nicht die Gabe, Gesehenes in seine Ursachen zu zerlegen, er konnte nur alles auf seine scharfen Sinne einwirken lassen, Erfahrungen nur insoweit sammeln, als sie sich an das anfügen ließen, was er zu suchen, was er zu meiden hatte“ (S. 7).

Wichtig scheint, dass durch diese Restriktion (wie schon oben) zwar die kognitive, nicht aber eine emotive Erlebnisfähigkeit des Bären beeinträchtigt wird. Das ontologische Bild, das in der Geschichte von dem Bären entsteht, insistiert auf einem Unterschied von Kognition und Bewusstsein Bär/Mensch, negiert diese aber bei ersterem nicht. Das wird auch am Ende der Geschichte bestätigt. Nach seiner lebensbedrohlichen Schussverletzung mühte sich „sein letztes Bewusstsein [...] vergeblich ab, die Ursache seiner Todeswunde zu finden“, doch als dieses letzte Bewusstsein verströmt, fühlte er „wie die volle Einsamkeit nahte, die er einst in seinen Bergwäldern genossen“ (S. 14-15). Eine meta-physische Ebene wird dem Bären implizit wie explizit zugestanden. Der Bär hat einen „Instinkt ähnlich jenem, der ihn unbekannte Gebiete durchforschen [lässt]“ (S. 11), er ist „Sucher von irgendetwas ihm Unbegreiflichen [...]" (S. 10). Diese Ebene der Metaphysik scheint eine eigene Emotionskategorie darzustellen.

Die Raumsemantik ist ein markantes Element, mit welchem wildtierliche und menschliche Emotionen erzeugt und strukturiert werden. Der Wald, darüber Berge 
und ihre Gipfel, auf denen blaue Heidelbeeren wachsen, die von gedämpftem Herbstsonnenschein umflossen werden und an die „der Tag voll Wellenschlag eines unendlichen Meeres [...] heranfloss [...]“ (S. 8), gehört zur Welt des Bären. Der Strom im Tal und die dortigen Straßen, Ortschaften, eine Stadt, sind Gebiet der Menschen. Die vertikale Abstufung der Topographie parallelisiert eine Degradierung bzw. Dichotomie der Emotionen: Unterhalb des Gipfels beginnt der Wald. „Hier war es ruhig [...], der Bach rauschte, Meisen und Finken zirpten in den Bäumen, Amseln raschelten im trockenen Bodenlaub, und ein Eichhörnchen ließ Bucheneckern hoch aus den Wipfeln fallen“ (S. 9). Im Tal fließt der Strom, „wie ein vom Feuer gekrümmtes Metallband durchschnitt er die Berge, die Wälder [... ““ (S. 8). Der mit den Menschen konnotierte Strom wird mit Feuer und schneidendem Metall metaphorisiert, mehr noch, es scheint ihm eine verschlingende Kraft innezuwohnen: Es schien, als „stürzten sie [die Berge] in ihn hinein, als ziehe sie der Strom tief in sich hinab“ (S. 8). Mit dem Auftakt des dumpfen Grollens, „dort wo der breite Strom die Berge durchschnitt“, leitet die Handlung die Überlappung der bisher weitestgehend getrennten Welten des Bären und der Menschen ein, denn „normalerweise kam der Bär nie so weit zu den großen Strassen der Menschen herab [...]“ (S. 7) und die Menschen waren in den Wäldern nur manchmal zu sehen. Nun aber „fanden [die Menschen] nicht mehr Platz auf der Straße, es war als schäumten sie über, stiegen über die Ufer gleich einem Strom, fluteten über die Wiesen [... “ (S. 11). Im Raum von Menschen und Tal nimmt der Bär - zum ersten Mal - eine Stadt wahr. Die Höhen um die Stadt „waren kahl, zerwaschen, zerfressen“. Die Emotion Ekel wird expressionistisch, sprachlich-ästhetisch von Architektur auf belebte und unbelebte Natur expandiert: Der Bär

„sah das ganze Tal durchsetzt mit eckigen Erhöhungen, die ihm wie Wucherungen waren, gleich den Auswüchsen am Stamme alter Bäume, wie Schorf einer Wunde, die nie heilte [...]. Und aus diesen Wucherungen heraus und hinein krochen Menschen gleich Maden am schon zerfallenen Kadaver eines Tieres" (S. 12).

Ein weiterer Faktor, der Emotionen unmittelbar sowie mittelbar mitbedingt, ist Bewegung ${ }^{(16)}$. Vom ersten bis zum letzten Satz der Geschichte findet Bewegung statt, am Anfang steigt der Bär (vom Berg) hinab, am Ende versinkt seine Kraft und verströmt sein Bewusstsein. Makroskopisch ist die Geschichte Bewegung. Der Vorgang des Bewegens wird mit Verben, Substantiven und Adjektiven eingeleitet, der Bär durchquert, wandert, das Licht durchfliesst die Welt der Gipfel, der Tag ist gleich eines Wellenschlages, Bär und Natur sind ruhig. Mit dem Ankommen der Schallwellen (Lärm) aus der menschlichen Welt, werden die menschlichen Bewegungen, die zugleich Flucht und Angetriebensein evozieren, als Kontrast über die Folie der Bärenwelt gelegt: „[...] aber etwas kam heran, unsichtbar und doch dem Tiere fühlbar, sich durch den Wald wälzend wie in

16 Siehe Wolfgang Hallet und Birgit Neumann (Hg.), Bewegung in der Literatur. Die Literaturwissenschaften und der Spatial Turn, Bielefeld, Transcript, 2009. Einen einschlägigen Verweis auf die Verbindung zwischen Bewegung und Emotionen (und Literatur) machte Jean-Marie Guyau, der davon ausging, dass Emotionen sowohl mittel- als unmittelbar, bewusst und unbewusst übertragen würden, und dass diese mit Bewegung gleichzusetzen seien: „Im Grunde gibt es nur Empfindungen von Bewegungen, und in jeder dieser Bewegungen kann man eine mehr oder minder elementare Nachahmung der wahrgenommenen Bewegung erblicken“, in: Koppenfels/Zumbach, Handbuch Literatur und Emotionen (Anm. 12), S. 485. 
einer wahnsinnigen Flucht“ (S. 8). Die menschlichen Gestalten fluten wie durcheinandergepeitschte Gegenstände, auf einer Strasse, die steigt und stürzt und sich quetscht, die Stadt frißt sich breit (vgl. S. 10). Am Ende ihrer Flucht sind die Menschen zusammengekrümmt, ihr Bild gleicht einer Choreographie des Leidens und des Schmerzes. Der Abscheu des Bären vor den Menschen wird nicht nur durch Geruch thematisiert, er bewegt sich auch, die Unruhe ist dem Bären lästig wie ein Mückenschwarm und in sein Lauschen und Fühlen „[...] krochen sie [die Menschen] hinein wie ekliges Gewürm [...]“ (S. 14). Alscher verwendet ein Bild, das das Moment der Bewegung innerhalb der dichotomen Raumsemantik von Bär und Mensch präsentiert. Bevor der Invasor Mensch den gewohnten Rhythmus des Bären stört, „wirkte das Treiben der Menschen nur wie ein ferner Widerhall, dessen Schwingungen [den Bären] nicht unmittelbar berührten “ (S. 7). Der Bär lebt in einer Welt, „die Berührungskreise [...] in sich fasst [...] (S. 9). Durch das Eindringen von Einflüssen aus der menschlichen Welt in diese „Berührungskreise“, movere, werden auch Emotionen „materialisiert“ bzw. transportiert. Kleinere Worteinheiten benennen und vermitteln mehr oder weniger direkt über Bewegung Emotionen. Mittelbar werden aber auch, in größeren Sprachsequenzen, Emotionen auf einer Metaebene konstruiert, die die Bewegungen in den Gesten, Ausdrücken, Kontakten, Höhenunterschieden der Landschaft, Dynamiken der Elemente wie Wasser und Wind, von Tönen, zwischen Bewusstsein und Unterbewusstsein, einschließt.

Ein weiteres konstitutives emotionales Moment dieser Geschichte sind psychoakustische Spektren. Laute sind sowohl intrinsisch emotionsgeladen als kulturell kodiert und können durch geschriebene Worte zu einem nachvollziehbaren Erleben gebracht werden - sei hier unterstellt. „Sowohl Musik als auch Literatur sind auditiv wahrzunehmen [...], erfolgen dynamisch, bewegen sich durch Abfolge von Worten oder Tönen [...]“(17). Wie bei der Raumsemantik ist die akustische Welt (von Bär/Mensch) dichotom. Es gibt die - hier stark dominanten - akustisch menschgemachten Phänomene, d.h. die Stadt mit ihren schrillen Klängen, die Strasse mit ächzenden Rädern sowie die menschlichen Laute; und es gibt die Klangwelt des Bärenuniversums mit dem Wald, in welchem der Bach rauscht, die Meisen zirpen und die Amseln im Laub rascheln. Während letztere Geräusche im GEMS-Modell ${ }^{(18)}$ mit Emotionen in den Zonen Friedlichkeit und Nostalgie auszumachen wären, tönen mit (Worten) der menschgemachten Geräuschkulisse entgegengesetzte, feindselige Emotionsskalen an. Lärm, Bersten, Krachen, Brausen, Rollen, Tosen usw. deckt sich mit der Beschallung einer Kriegsmaschinerie, die adjektivisch und adverbial amplifiziert wird und von einem unharmonischen Rhythmus begleitet wird:

„An diesem Morgen, da klar und fernenfrei der Tag kam und der Bär auf einem Gipfel langsam zwischen den Felsen herumschlenderte, hob ein Dröhnen, Rollen und Prasseln

17 Karin HochradL, Olga Neuwirths und Elfriede Jelineks gemeinsames Musiktheaterschaffen. Ästhetik, Libretto, Analyse, Rezeption, Bern, Peter Lang, 2010. Siehe „Although being perceptually non-realistic, emotional content in verbal stimuli can evoke strong and reliable emotional responses on behavioral and brain level engaging manifold brain networks [...]", in: Lorna Schlochtermeier, Experiencing Emotions in Different Media Types: Psychological Processes and Neuronal Correlates, unveröffentlichte Dissertation, FU Berlin, 2016, www.diss.fuberlin.de/diss/servlets/MCRFileNodeServlet/FUDISS_ derivate_000000019922/DissertationSchlochtermeier_Online.pdf. 
am Strome an. Schwere Schläge erschütterten die Luft, sie folgten einander immer schneller, bis nichts mehr war als ein ununterbrochenes Schüttern und Brausen [...]“ (S. 8).

Selbst der Unhörbarkeit der bedrohlichen Atmosphäre gibt Alscher mit der Einbindung von sonoren Metaphern Ausdruck:

„Es wurde Nacht. Etwas wie ein atemloses Keuchen, wie Schreckensschreie war über den Wald gebreitet. Oft, wenn der Bär auf eine kahle Höhe kam, richtete er sich hoch auf und lauschte hinaus in die Weite, in der ein Brausen, ein Rauschen der Nacht herrschte, als werde diese tief von innen durchwühlt" (S. 9) ${ }^{(19)}$.

Die physiologischen Laute, die die Menschen von sich geben, sind durchwegs negativ besetzt und präsentieren über die Basiskategorien Angst und Traurigkeit hinausgehende Emotionen der Desolation, des Leidens, welche in ihrer Valenz durch die Evokationskraft von Urlauten als gesteigert bewertet werden dürfen. „Heisere, müde Stimmen klangen, eintönig, manchmal schmerzlich aufschreiend“ (S. 13); von vor Stöhnen erfüllten Straßen, von Notschreien, Angstrufen und von Keuchen ist die Rede. Allerdings werden diese Emotionen durch die Handlung potentiell abgeschwächt bzw. modifiziert: Der Text evoziert nämlich die Eigenverantwortlichkeit des Menschen für das im Stöhnen ausgedrückte Leiden. Mehr noch, der Plot, in dem die (verdiente) Sehnsucht des Bären nach Ruhe und Frieden gestört wurde, zeichnet den Bären in der Perspektive des Opfers jener Menschen. Als der Bär sich „in leidenschaftlichem Drange zurück [-wandte,...] um seine Wälder wieder zu erreichen“ hört er zwei Schüsse und ist - unnötiges, ungerechtfertigtes - Opfer der Menschen geworden. Der Bär, der zuvor nur lauschte, erhebt zum ersten und letzten Mal seine Stimme und „brüllte wild auf“ (S. 14). Dieses Aufbrüllen kontrapunktiert sein Sterben und invertiert dabei eine Komponente des menschlichen Schreiens in seiner moralischen Note.

Der Wolf ist in selbiger Geschichte ein „liminal animal“(20), das - anders als der Bär - den Raum des mit dem Bären konnotierten Wildtieres verlassen hat. Er wird als Spiegelbild des Menschen, des homo homini lupus, gezeichnet und ist Zeichen des Schlechten im Menschen. Anders als der Bär, der zwar auch versucht ist, den Menschen in der Bewegung der Flucht zu folgen, sich ihnen anzunähern, seinem Instinkt unterliegend sich von deren Angst animieren zu lassen und seinen Blutdurst und Hunger an ihnen zu stillen, den aber „der Geruch von Schweiß, Erschöpfung, von abgehetztem Blut [...], der Geruch der Verwesung [...]“ (S. 13) abstößt, unterschreiten die Wölfe jene Distanz zu den Menschen, „rissen Haustiere, balgten sich um gefallenes Vieh, schnappten wütend eifersüchtig nach Geiern und Raben [...]. Sie waren mit Blut und dem Inhalte der Eingeweide beschmiert und stanken nach verwestem Fleische [...]" (S. 11). Menschen werden im Zusammenhang des desolaten Kontexts als verroht beschrieben, dieser Eindruck wird mit der Nivellierung auf die Wolfs- bzw. Tierebene erzeugt und in der perspektivischen Lenkung über die Reflexion des Bären akzentuiert. „Und dann kamen die Flüchtenden [...], die kümmerten sich nicht um den Notschrei der anderen [...]. Der Bär aber erwartete noch, dass sie über ihre verwundeten und sterbenden Kameraden herfallen würden, wie es die Wölfe taten“"(S. 12).

19 Kriegsgeräusche sind ein naturwissenschaftlich untersuchtes und poetisch nicht unberührtes Feld. Siehe Reinhard Osтекотн, „Das große Dröhnen“, Die Zeit, 18.02.2016.

20 R. Borgards, Tiere (Anm. 1), S. 83. 
Eine Versuchsanordnung wie bei Ellsworth, Fontaine et al., bei der Probanden den in Fragmente zerlegten Text dieser Geschichte, sortiert in Unterteile, die auf Menschen („Fluten durcheinander gepeitschter, willenloser Gegenstände“, „Kümmerte sich nicht um den Notschrei der anderen“, „Stumpf und willenlos“, usf.), auf den Bären („Unsichtbar und doch dem Tiere fühlbar“, „Ohne Scheu“, „Alleinseinwollens“, usf.) und auf den Raum („Blaue Heidelbeere“, „Krachen und Bersten“, „Dunst und gelbliche Schwaden“, usf.) bezogen sind, würde bestätigen, dass sich die o.g. vier emotionalen Dimensionen bzw. die sechs emotionalen Komponenten wiederfinden: Die Bewertungsdimension (a) ist dominant und würde bei den Menschen negativ, beim Bären positiv ausfallen, die landschaftlichen Attributiva bei der Kategorie Mensch wären negativ, respektive positiv beim Bären. Handlungstendenzen gehen daraus hervor, wie z.B. der Bär, der sich wegen des Leichengeruchs von den Menschen abwendet. Die arousal-Dimension (b) ist notwendig, um psychophysische Zustände der Charaktere einzufangen und die Handlung zu plausibilisieren. Ebenso ist die Kontrolldimension (c) detektierbar, der Bär, der frei über Wiesen und Wälder schreitet, die Ohnmacht der Menschen dagegen, die hilflos sind und flüchten, die sogleich Täter und Opfer des Krieges sind. Auch die Dimension des Neuen (d) ist vonnöten, um beispielsweise den Handlungsumschwung vom Idyll zur Bedrohung des Friedens zu lenken oder das plötzliche Vorspringen der Menschen, die den Bären erlegen, einzuordnen.

Geht man von der Schöpfungsthese literarischer Texte aus, so hört die Gestaltung und Kreierung von Emotionen - auch im Verhältnis Mensch und Wildtier - über Bilder, Wortschöpfungen, Genres, Narrative usw. erst an den Grenzen der Kunst auf. Im Folgenden soll nun die Frage lauten, nach welchen Emotionen gefragt werden muss, um in Alschers Geschichten etwas sehr Spezifisches zwischen Menschen und Wildtieren einzufangen. In der Repertorisierung der Emotionen in Über den Menschen sticht eine Häufung der Emotionen Ekel, Erhabenheit und Schuld bzw. Gerechtigkeit hervor, Emotionen, die eine Affinität zu sog. moralischen Emotionen haben.

\section{Moralische Emotionen}

Mit Ekel, Stolz, Verachtung, Scham, (Un-)Schuld(igkeit) sind in Alschers Geschichten Emotionen, die unter die Kategorie moralisch fallen (oben unter den 24 prototypischen Emotionen $\mathrm{zu}$ finden) vertreten; sie sind $\mathrm{zu}$ unterscheiden von nicht-moralischen Freude- oder Ärgergefühlen. Hinzuzufügen wären allerdings u.a. Reue, Bewunderung, Respekt, Gerechtigkeit und Schadenfreude. Moralische Emotionen, weit verbreitet in Wirklichkeit und in Literatur, bilden eine Mittlerfunktion zwischen Kognition und Verhalten und dirigieren nicht nur die Urteilsebene, sondern sind auch ein Motor für ein moralisches Handeln. Moralische Emotionen setzen relativ einfache kognitive Fähigkeiten voraus und sind schon von Kindern erlebnisfähig ${ }^{(21)}$. Ein abstrahierter Blick auf moralische Emotionen erlaubt eine Einteilung in positiv und negativ, und in auf sich selbst bezogene und auf Andere gerichtete moralische

21 Katrin Schulz, Moralische Emotionen, unveröffentlichte Dissertation, TU Chemnitz, 2011, S. 24. Es gibt auch Stimmen, die Tieren moralische Emotionen wie Gerechtigkeit nicht kategorisch absprechen: Marc Bekoff und Jessica Pierce, Sind Tiere die besseren Menschen?, Stuttgart, Kosmos, 2017. 
Emotionen, self-directed/self-conscious und other-directed emotions ${ }^{(22)}$. Und es ist ein Unterschied, ob es um moralische Emotionen des Beobachtenden - und der Leser ist nicht zuletzt Beobachter - oder des Handelnden geht, da der perspektivische Standpunkt die Valenz moralischer Handlungen beeinflusst; geht es um sich selber, liegt die Tendenz vor, die Handlung nach ihren Konsequenzen zu beurteilen, geht es aber um Andere, ist man geneigt, deren Handlungen eher an übergeordneten moralischen Leitlinien zu messen ${ }^{(23)}$.

In Alschers Geschichte Die Fremde wird in der De-Komposition des Handlungsstrangs aus moralischen Emotionen deren innerer Einfluss auf die Akteure sowie auf ihr Verhalten evident. Ein Erzähler in der 3. Person ist unbeteiligter Beobachter der Handlung und lässt die direkte Rede der Akteure zu. Hauptfigur ist ein Junge, Nebenfiguren Bruder und Vater. Handlungsrahmen ist ein Besuch des Jungen in dessen Ferien in den Bergen, wo Vater und Bruder Schafhirten sind. Ein Wolf ist das Objekt (im Text), das die gemischten moralischen Emotionen anzieht. Implizit werden diesem Wolf self-conscious emotions zugestanden. Anfänglich labile positive auf den Wolf gerichtete Emotionen verwandeln sich schnell in die negativen moralischen Emotionen Geringschätzung und Schadenfreude, bevor sie schließlich wieder übergehen in Bewunderung und Ehrfurcht. Es gibt in der Handlung zwei übergeordnete moralische „Instanzen“, die eine Spannung zum emotional-moralischen Innenleben der Figuren bilden. Die eine ist das Schulwissen:

„Es fiel ihm wieder ein, was er in der Schule vom Wolf gelernt hatte. ,Der Wolf ist ein Raubtier, das sich tief in Wäldern verborgen hält, das nachts umherstreift, Menschen und Tiere anfällt, des Winters, von Hunger getrieben, weite Wanderungen unternimmt und dann sehr gefährlich wird.' Ja, das hatten sie gelernt" (S. 78).

Die andere ist in Person des Vaters, der dem Wolf Respekt auf nahezu gleicher Augenhöhe billigt: „Der Wolf leidet es eben nicht, dass wir uns in seinem Walde aufführen als wären wir die Herren“ (S. 83). Die erste reale Begegnung mit dem Wolf ist ein distanzierter Blickkontakt und ist gleichzeitig faszinierend und verwirrend für den Jungen. Das (negative) Schulwissen über den Wolf als kognitive Komponente und demgegenüber die Präsenz des Wolfes „[...] so stark und frei auf einem Gipfel in der Ferne [...]“ (S. 78), die eine (positive) affektive Komponente auslöst, widersprechen sich. Die moralische Entwicklung beginnt, indem Alscher die auf den Wolf gerichtete emotionale Geringschätzung siegen lässt und diese mit der Emotion Schadenfreude anreichert. In seiner Phantasie sieht der Junge „wie der Wolf an ihm vorbei will, [er] aber schlägt plötzlich mit der Axt auf den Stamm, da duckt sich der Wolf, prallt seitwärts und jagt dann voll Angst in den Wald hinein, mit jähen Sätzen und eingezogenen Flanken“ (S. 80). Geringschätzung und Schadenfreude geben Alscher Gelegenheit eine motivationale Komponente einzubringen: die Tötung des Wolfes. Der Erzähler schlägt eine Möglichkeit vor, wie es zu diesem emotionalen Umschlagen gekommen sein könnte:

22 Erweiterungsfähig durch die Kategorien other condemning-emotions, other-suffering emotions und other-praising emotions. Siehe Johannes FISCHER und Stefan GRUndEN (Hg.), Die Struktur der moralischen Orientierung. Interdisziplinäre Perspektiven, München (u.a.), LIT Verlag, 2010, S. 102-103. 
„Vielleicht war der Hass dieses Knaben aus dem unbewussten Gefühl jener Beschämung entstanden, dass er es nicht vermocht hatte, den Wolf in die Flucht zu treiben, sondern durch seine Erscheinung wie gelähmt wurde und erbittert, weil der Wolf so unbekümmert an ihm vorbeilief" (S. 83-84).

Die narrative Umsetzung der Motivation gestaltet sich in der Stellung einer tödlichen Falle für den Wolf. Der Junge und sein Bruder installieren im Wald einen Köder (Tribut leisten Kadaverstücke eines Hundes, der den Wolf in seinen Wald verfolgte und von diesem getötet wurde) mit dem der Wolf einen fatalen Selbstschuss auslösen soll. Die Bemühungen bleiben allerdings vergeblich, nur ein Fuchs geht anstelle des Wolfes in die Falle. „Dieses vergebliche Bemühen ließ den Knaben den Wolf nur noch mehr hassen. Es war etwas Schmerzliches in seinem Hasse, allmählich kam aber Bitterkeit dazu [...]“ (S. 85). Nach Fritz Heiders Konzept der naiven Handlungstheorie kann man einen Zusammenhang zwischen der Entstehung von moralischen Emotionen und folgenden Komponenten herstellen: Die übergeordnete Valenz einer Situation, das Sollen; ob dieses Sollen erreicht wird; das Kriterium der Anstrengung, des Aufwandes. Letztere seien ausschlaggebend zur Bewertung des Grades der Verantwortlichkeit der Erreichung des Ziels einer Person ${ }^{(24)}$. Der Junge investiert viel Zeit und Hoffnung in den Versuch, den Wolf zu töten, und dies untermauert seine Verantwortlichkeit. Demgegenüber steht als kodiertes kulturelles Wissen, dass Töten schlecht ist. Dieser Orientierung entspricht die höhere moralische Instanz des Vaters, der das Recht des Wolfes auf Leben explizit bejaht und - wie gleich eingangs der Geschichte mitgeteilt wird - der ein scharfes Gesicht hat und dessen Augen durchdringend blicken, wodurch implizit etwas wie Wahrheit konnotiert wird und also seine moralische Integrität unterstrichen wird. Zudem scheint sich durch die hohe Anstrengung der „kathartische moralische Effekt“ zu erhöhen. Als der Junge den Wolf - der offensichtlich versucht hat, ein Schaf zu rauben und dabei in der Hütte eingesperrt wurde - in einer anderen, unerwarteten Falle vorfindet, sind sein Spiegel der Geringschätzung und sein motivationaler Impuls, den Wolf zu töten, sehr hoch. „Ein Schuss mit der Pistole durch das Fenster, und die Bestie wälzt sich im Blute. [...] Schreiend, schimpfend rief der Knabe den Wolf an“ (S. 87). Das narrative Detail, über welches die Wende in der human-animal-gerichteten Emotion beschrieben und ausgedrückt wird, ist der Blick ${ }^{(25)}$. Einem physischen, räumlichen und metaphysischen Erlebnis gleicht

24 Ebd., S. 10-11.

25 A. Goodbody, „The Hunter as Nature-Lover“ (Anm. 4), S. 149. Die Explizierung der Bedeutung des Blicks für die Begegnung, für die Problematik der Differenzierung, für die Phänomenologie MenschTier, bleibt an dieser Stelle begrenzt. Als ein inhaltlicher Bestandteil von Alschers Geschichte(n) verweist er, der Blick, auf die Relevanz der Begegnung der Augen bei der Bildung oder Aushandlung moralischer Emotionen zwischen Mensch und Wildtier: Jacques DerRidA, L'animal que donc je suis, Paris, Éditions Galilée, 2006. Siehe „Das Tier beobachtet ihn [den Menschen] genau, über einen schmalen Abgrund des Nicht-Verstehens hinweg. Aus diesem Grund kann der Mensch das Tier überraschen. Der Mensch blickt ebenfalls über einen ähnlichen, wenn nicht identischen Abgrund des Nicht-Verstehens hinweg. Wo immer er auch hinblickt. Er blickt immer über einen Abgrund aus Unwissenheit und Angst. Wenn er nun von einem Tier wahrgenommen wird, wird er so gesehen, wie er seine Umgebung sieht. Weil er dies erkennen kann, wird ihm der Blick des Tieres zu einem vertrauten“, in: John Berger, Das Leben der Bilder oder die Kunst des Sehens, Berlin, Wagenbach, 1989, S. 15. 
dieser Augenblick der gegenseitigen Begegnung von Mensch und Wildtier, in dem das Wesentliche der Emotionen kondensiert zu sein scheint.

„Und was wollte dieser Blick, dieser unerklärliche, glimmende Blick von ihm, der an ihm riss und ihn doch wieder fortstieß? Es war ihm plötzlich, als stehe er in einer offenen Tür, als gäbe es keine Schutzwand zwischen ihm und dem Wolf [...]" (S. 88).

„Seine [des Jungen] [...] Augen wurden trübe, wie durch einen Nebel fühlten sie den regungslosen Blick des Tieres, umfassten die machtvolle starke Gestalt, die in diesem kläglich engen Raum so ungeheuer erschien, als stieße sie an die Decke [... ““ (S. 87).

Die Emotion, die in der Sequenz des Blickkontaktes präsentiert wird, hat wohl mit Moral zu tun, denn in der Handlung geht es um die Macht des Tötens versus das Lebenlassen. Dies berührt übergreifend das Thema Sterben und Tod. In einem Gedanken blitzt, zum letzten Mal, das Verlangen des Jungen auf, den Wolf zu töten, mit einem Knüppel, aber dann kam es „ihm so unglaublich vor, dass dieses Tier getötet werden konnte [...], unbeweglich und leblos daliegen könne [...], dass diese Augen sich im Tode verändern könnten [...]“ (S. 88). Der Anblick des gefangenen Wolfes, der getötet werden könnte, interferiert mit der unbewussten Angst (Angst als moralische Emotion) vor dem eigenen Tod, der sozusagen verdrängt (oder überwunden) wird mit der intuitiven Entscheidung, dem Wolf das Leben zu schenken. „Aber wozu stand er denn vor der Türe und hielt sie verschlossen? Er musste sie ja öffnen, musste sich selbst den Weg frei machen, der ihn von dieser Macht da drinnen schied, oder er müsste selbst zusammensinken, würde verlöschen“ (S. 88). Am Ende verschwindet der Wolf, „es hatten ihn wohl die Felsen auf dem Gipfel aufgenommen [... “ (S. 89). Die Lösung des moralischen Konflikts (bei der eigentliches Mitleid nicht eingesetzt wird) gelingt in der erfolgreichen Transmutation von geringschätzigem Hass in Ehrfurcht.

\section{Existentielle Emotionen}

Die Geschichte Die Tiere ernten endet mit der Tötung eines Menschen durch einen Wolf, ersterer wird zerfleischt, das Töten wird prägnant über die die Szene begleitende an- und abebbende Geräuschkulisse des Widerstands, des Fressvorgangs und den Regungen der Agonie beschrieben: „[Der Wolf stürzte sich] mit einem Sprung auf ihn. Und dann war nichts als einige heisere Laute, ein Knirschen, ein Zerren, ein Schlagen im Laub und langsam kommende Stille“ (S. 95). Die moralische Emotion Ungerechtigkeit bzw. Schuld, die in der Isolierung dieser Sequenz und der Information, dass das Opfer verletzt ist, entstehen könnte - man denke an Über den Menschen, wo der Bär in einer vergleichbaren Situation von Hunger und Überlegenheit den Menschen am Leben lässt, der Bär aber von Menschen unnötig getötet wird - ergibt sich jedoch im Zusammenhang kaum. Zum einen hängt ein strukturelles Element damit zusammen: Erzähltechnisch gibt der Sprecher in dieser Geschichte die Wahrnehmung der Welt und die impliziten Emotionen, die die Hauptfigur, den Wolf, mit ihr verbinden, ausschließlich über dessen Perspektive wider ${ }^{(26)}$. Durch die dadurch graduelle Abschwächung der anthropozentrischen Perspektive wird das intrinsische Recht des Wolfes

26 Alschers Geschichten könnte man (ähnlich wie z.B. bei Felix Salten) als biozentrische Werke bezeichnen. Summa summarum ist die Anzahl, insbesondere der nicht-domestizierten nicht-menschlichen Tiere, den menschlichen Tieren deutlich überlegen, wodurch potentiell eine bioethische Orientierung favorisiert wird. 
auf Leben und auf Fressen gestärkt. Der Wolf erscheint eher als Subjekt, der Mensch als Objekt. Zum anderen ist in dieser Geschichte aber eine Emotion im Spiel, die die moralische Emotion zu beeinflussen (und zu dominieren) scheint. Sie kann als eine eigene Dimension, als existentielle Emotion entschlüsselt werden. Das Zugeständnis der Erlebnisfähigkeit einer solchen Emotion an nicht-menschliche Tiere, auch wenn sie mit der menschlichen nicht identisch ist, erhöht weitreichend deren Status und ein ebenbürtiges Anrecht auf Vertretung eigener Interessen, was neben der o.g. Perspektivierung das Töten des Wolfes moralisch neutralisiert. Matthew Ratcliffe versteht unter existentiellen Emotionen solche, die nicht an eine spezifische Situation oder ein Objekt gebunden sind.

„The world as a whole can sometimes appear unfamiliar, unreal, distant or close. It can be something that one feels apart from or at one with. One can feel in control of one's overall situation or overwhelmed by it. One can feel like a participant in the world or like a detached, estranged observer staring at objects that do not feel quite, there ${ }^{\text {c (27). }}$

Getrennt zu sehen sind diese Emotionen von der atmosphärischen Komponente, deren Beschreibung auf das Außerhalb fokussiert ist. Existentielle Emotionen können entweder eine Beziehung zu sich selbst (self-conscious) oder zu (den) anderen Wesen oder zur Welt als Ganzes haben, oder eine kombinierte Beziehung zu allen diesen Ebenen haben. Alscher beschreibt die Umwelt und deren atmosphärische Schattierungen ausgiebigst, implizit und explizit. Darüber hinaus verleiht er Tieren wie diesem Wolf aber auch ein existentiell bewusstes Erleben der Welt. Der Wolf „[...] beherrschte den Lufthauch, der die Waldhänge der Schlucht hinauf- oder hinabstrich. Er konnte ihn durchforschen [...]“ (S. 91), und er reagiert auf Veränderungen, die sein Seinsgefühl, das Gefühl seines Da-Seins, beeinträchtigen. Viele der Alscher'schen Wildtiere erleben bewusst fühlend die eigene Vitalität und Freiheit, und meist fungiert der Mensch dabei als Antagonist. Passagen wie diese,

„Einsam und unberührt war die Wildnis wie vordem, trotzdem aber klaffte ein Riss durch die Stille. Etwas in ihr war zerstört, hatte sich schrecklich geändert, und so tief die Ruhe des Urwaldes auch war, so friedlich ihr Weben, ein Schrillen durchzitterte sie, schnitt in sie hinein, so dass sie der Bär schmerzhaft wie eine Wunde empfand“ (Zerstörung, S. 108).

„Diese Ferne der Erde, dieses Luftmeer, das ihn [ein Adler] von ihr trennte, welches er zu verkleinern oder zu vergrößern vermochte, wie er wollte, war ihm die tragende Kraft, das Bewusstsein seines Wesens, jenes Herrschergefühls über Welt und Tiefe und allem, was sich dort unten bewegte" (Der gestürzte Adler, S. 55).

„Der Wolf fühlte sich plötzlich seinem Walde fremd geworden“ (Verfolgter, S. 104).

präsentieren und thematisieren Seinsgefühle, die nicht unmittelbar auf ein konkretes Vorkommnis gerichtet sind und drücken, in den Worten Ratcliffes aus, „how we find ourselves in the world [... “ ${ }^{\text {(28) }}$. Ein Bewusstsein, ein Selbst-Sinn, ist eine Voraussetzung für diese Art phänomenalen Erlebens, für welches nach Eva-Maria Engelen wiederum ein Körpergefühl vorhanden sein muss: „Die Erfahrung des Körpers wird hier zur Bedingung des Bewusstseins, der Empfindungsraum Körper zur Keimzelle

27 Achim Stернал, „Existentielle Gefühle und Emotionen. Intentionalität und Regulierbarkeit“, in: Jörg Fingerhut und Sabine Marienberg (Hg.), Feelings of Being Alive, Berlin/Boston, De Gruyter, 2012, S. 109-110. 
des Selbstsinns“(29). Mit Alschers Geschichte der Mensch und die Frage an die Erde kann man eine feine Linie ziehen zwischen der Ontologie von Tier und Mensch. Der Mensch in dieser Geschichte formuliert in seinem Delirium - ein Zustand der zugleich eine existentielle Emotion und eine Körpererfahrung ist - das Dilemma menschlichen Seins als die Verdammnis zur Metaphysik ${ }^{(30)}$. Er begreift das „Menschentum [als den] erschütternde[n] Kampf eines schwachen Leibes mit einem überstarken Geiste [...]“ (S. 113), den Menschen als „entartetes Tier“ (S. 112), wobei nicht das Tier, sondern die Entartung pejorativ ist ${ }^{(31)}$.

Hinter der subtilen Hineinprojizierung der Dimension der existentiellen Emotionen in manche Wildtiere verbirgt sich bei Alscher mehr als hinter allen anderen Emotionen: eine Art hierarchischer Einstand von Wildtier und Mensch. Ein geringer aber entscheidender Unterschied macht, dass das nicht-menschliche Wesen nicht von einem metaphysischen Sog eingezogen wird, differenziert so wiederum Mensch und Wildtier und gibt darin zugleich dem Wunsch Ausdruck, zu sein wie ein Wildtier. „Und weil er an seinem Menschentum verzweifelte, wollte der Einsame Tier sein. Wild und ursprünglich und voll Größe der unverbrauchten Kraft wollte er sein. [...] Wie ein Raubtier verließ der Einsame nachts seine Höhle“ (S. 112) ${ }^{(32)}$.

Mit wenigen Ausnahmen findet die Handlung Alschers Geschichten in Lebensräumen statt, die nicht gänzlich von der Zivilisation vereinnahmt sind. Wie der etymologisch bereits hergestellte Konnex von Bewegung und Emotion bereits andeutet, bewegen Emotionen die Handlung. Ohne Emotionen in Menschen und Wildtieren kämen die Narrative nicht zustande. Die Wahrscheinlichkeit eines Konflikts zwischen

29 Eva-Maria Engelen, Gefühle, Stuttgart, Reclam, 2012, S. 75.

30 „Statt primär rein ,ontisch“ auf die konkrete Welt bezogen zu sein, deren Realität erst noch fraglos gewiss scheint, ist das menschliche Dasein immer schon auch, ontologisch ' auf das Sein der Welt und das eigene In-der-Welt-sein als solches bezogen. Dieser doppelte, nämlich ontische und ontologische Bezug zu Welt und Selbst gehört zum menschlichen Existieren und gefährdet es zugleich. Die Gefährdung liegt darin, dass der scheinbar fraglose Seinsglaube jederzeit schwinden kann. Denn während bei Husserl erst der Philosoph durch einen bewussten Willensakt den Seinsglauben einklammert und damit von der naiv-natürlichen in die reflektiert-philosophische Einstellung wechselt, existiert der Mensch bei Heidegger immer schon unfreiwillig philosophisch, ist ,Metaphysik' [...]“: Alice Holzhey-Kunz, „Das Gefühl des Lebendigseins ist ontisch-ontologisch zweideutig“, in: FingERHUT/ Marienberg, Feelings of Being Alive (Anm. 27), S. 132.

31 Heidegger begreift das Tier (gegenüber dem Menschen) als different, insofern „[...] dem Menschen das Seiende als solches im Ganzen zugänglich ist, [...] er Welt [hat] bzw. lebt in einer Welt. Dem Tier [...] das Seiende als solches hingegen nicht zugänglich [ist]“. Heideggers „Tier“ steht „immerhin in einer Beziehung zu dem es Umgebenden“; aber es [das Tier] ist „weltarm“. Alschers Wildtiere aber sind mit einem ebenbürtigen Sinn für das Seiende und das eigene Dasein ausgestattet und neben ihnen erscheint der Mensch oft als weltarm. Siehe Friederike RESE, „Horizontbildung und Weiterbildung. Zur Mensch-Tier-Differenz in Heideggers Grundbegriffe der Metaphysik-Vorlesung“, in: Babette Babich, Alfred Denker und Holger Zoborowski (Hg.), Heidegger \& Nietzsche, Amsterdam/New York, Rodopi, 2012, S. 403.

32 Eventuell verbirgt sich hinter dem Topos des Tier-Werdens weniger das Ziel der totalen Aufgabe des Menschen, eine Transformation von humanimal $\mathrm{zu}$ animal, sondern der Wunsch nach einer Mitte. „,Becoming animal' is therefore not an attempt to ultimately become the animal itself, but to try and understand the animal from the middle of one's relation with it; from the middle of difference [...]": Harry Wels, „Whispering Empathy: Transdisciplinary Reflections on Research Methodology“, in: Bert Musschenga und Anton Van Harskamp (Hg.), What Makes Us Moral? On the Capacities and Conditions for being Moral, Heidelberg/New York, Springer, 2013, S. 160. 
Wildtieren und Menschen wächst, je näher sich deren physische Lebensräume kommen und wenn sich die Interessen des Einen nicht mit denen des Anderen decken ${ }^{(33)}$. In solchen Situationen kommen moralische Emotionen ins Spiel. Sie sind maßgeblich daran beteiligt, wie Entscheidungen innerhalb eines Handlungsspielraums ausfallen. Bei Alscher heißt dies oft, bei Entscheidungen die intrinsischen Interessen des Anderen, des Wildtiers, zu begreifen. Die Entscheidungsphase, in der Gewissen zu einer moralischen Entscheidung, einem ethischen Verhalten reift, ist typischerweise mit einer einfühlenden Perzeption umgesetzt, in der das Andere dem Ich nahekommt ${ }^{(34)}$. In dieser Zone des Nahseins sind die Emotionen von Mensch und Wildtier humanimal, sie gehören zusammen ${ }^{(35)}$.

Die Analyse zeigt, dass es möglich und aufschlussreich sein kann, den Einstieg in ein literarisches Narrativ über Emotionen zu nehmen. Für Umweltverhalten und Umwelthaltungen ist die Bedeutung der Emotionen seit vielen Jahrzehnten anerkannt $^{(36)}$. Der Beitrag ist ein Beispiel, wie Wildtiere und Emotionen Ausgangspunkt sowohl der literarischen Animal studies als der Environmental studies sein können. Eine Kooperation von Literaturwissenschaft und Psychologie erweist sich als fruchtbar, da die psychologischen Methoden Objektivierung fördern. Angesichts des Umstands, dass es größere individuelle Schwankungen von Emotionen zu Wildtieren geben kann, erscheint dies wertvoll. Während die Forschung über die Geschichte der Emotionen mit Haustieren innerhalb der Animal studies sehr aktiv war und ist ${ }^{(37)}$, trifft dies weniger auf Wildtiere zu. Das hängt wohl damit zusammen, dass „Stories dealing with hunting and wild animals occupy a marginal status in twentieth-century German literature [... “(38). Dies spricht aber umso mehr dafür, einen Schriftsteller wie Alscher aus dem Schattendasein zu rücken und den Wildtieren in seinen Werken einen zentralen Platz als Akteure einzuräumen.

33 Dieser Umstand ist gültig weit über Alschers Werk hinaus. Dass die Verdrängung des „Wilden“ durch Mensch und Zivilisation gerade um die große Jahrhundertwende kritisch wahrgenommen wurde, davon zeugen nicht zuletzt neoromantische Werke bzw. eine Hinwendung zum Exotischen (in Reiseliteratur). Siehe Aurélie Choné, „Le message de l'animal dans Indienfahrt de Waldemar Bonsels“, in: Aurélie Choné und Catherine Repussard (Hg.), Des animaux et des hommes / Von Tieren und Menschen, Recherches germaniques, Sonderheft 10 (2015), S. 129-148.

34 Phylogenetisch sind empathische Vorgänge, nicht nur zwischen Menschen, auch in InterspeziesBeziehungen von Tieren und Menschen, tief verankert. Für Lori Gruen gibt es verschiedene Herangehensweisen an Empathie, u.a. versteht sie darunter den Modus einer moralischen Wahrnehmung: „Empathy is a particular type of attention, what I think of as a kind of moral perception. Moral perception is not the same as ordinary sense perception, in that the latter doesn't often require reflection and correction, whereas moral perception does." Siehe Lori Gruen, Entangled Empathy, New York, Lantern Books, 2015, S. 39.

35 Mensch und Wildtier sind mit den Worten Erica Gittins nicht mehr „monolithic“: Erica GitTINs, „The Archeology of Becoming the Human Animal“, Society \& Animals, 21 (2013), S. 120-133.

36 Elisabeth Kals und Markus Müller, „Emotions and Environment“, in: Susan Clayton, Peter Nathan (Hg.), The Oxford Handbook of Environmental and Conservation Psychology, Oxford, Oxford University Press, 2012, S. 128-147.

37 Pascal Eıtler, „Weil sie fühlen, was wir fühlen. Menschen, Tiere und die Genealogie der Emotionen im 19. Jahrhundert“, in: Gesine KrÜGer und Aline Steinbrecher, Tierische (Ge)Fährten, Wien (u.a.), Böhlau, 2011, S. 221-228.

38 A. Goodbody, „The Hunter as Nature-Lover“ (Anm. 4), S. 144. 


\section{Zusammenfassung}

Wildtiere nehmen in dem Geschichtenband Die Bärin von Otto Alscher einen zentralen Platz ein und Emotionen von und zu diesen Tieren spielen dabei eine bedeutende Rolle. Ausgehend von einer Studie der Psychologie, die zeigt, dass mehrere Dimensionen von Emotionen zum Erschließen von Emotionen in Wörtern oder Satzteilen nötig sind, analysiert der Aufsatz die sprachliche Konstruktion von Emotionen in Alschers Geschichten. Hierbei konzentriert er sich besonders auf die raumsemantische, kinetische und psychoakustische Gestaltung. Des Weiteren vertieft die Textanalyse die herausragende Rolle von moralischen und existentiellen Emotionen. Das Resultat ist eine Gleichwertigkeit von menschlicher und tierlicher Perspektive und eine Annäherung, wenn nicht Verschmelzung, der Emotionen von Menschen und Wildtieren.

\section{Résumé}

Les animaux sauvages occupent une place centrale dans le recueil d'histoires Die Bärin d'Otto Alscher, et les émotions, de et envers ces animaux, y jouent un rôle essentiel. Partant d'une étude en psychologie qui démontre la nécessité de recourir à plusieurs dimensions d'émotions pour comprendre celles qui se dégagent des mots ou des fragments de phrases, cet article analyse la construction linguistique des émotions dans les textes d'Otto Alscher. Il s'intéresse en particulier à la représentation de l'espace, des mouvements et de l'acoustique. En outre, l'analyse textuelle approfondit le rôle primordial des émotions morales et existentielles. Il en ressort une équivalence de la perspective humaine et animale et un rapprochement, voire une fusion des émotions des hommes et des animaux sauvages. 